\title{
Comparison between the KOMPSAT-1 drag derived density and the MSISE model density during strong solar and/or geomagnetic activities
}

\author{
J. Park ${ }^{1,2 *}$, Y.-J. Moon ${ }^{3}$, K.-H. Kim ${ }^{1}$, K.-S. Cho ${ }^{1}$, H.-D. Kim ${ }^{4}$, Y.-S. Kwak ${ }^{1}$, Y.-H. Kim ${ }^{1}$, Y.-D. Park ${ }^{1}$, and Y. Yi ${ }^{2}$ \\ ${ }^{1}$ Korea Astronomy and Space Science Institute, Hwaamdong, Yuseong-Gu, Daejeon 305-348, Korea \\ ${ }^{2}$ Dept. of Astronomy and Space Science, Chung-nam National University, Daejeon 305-764, Korea \\ ${ }^{3}$ Department of Astronomy and Space Science, Kyung Hee University, Yongin 446-701, Korea \\ ${ }^{4}$ Korea Aerospace Research Institute, Daejon 305-333, Korea
}

(Received June 25, 2007; Revised November 12, 2007; Accepted December 23, 2007; Online published July 4, 2008)

\begin{abstract}
We have compared the KOrea Multi-Purpose SATellite-1 (KOMPSAT-1) drag derived density with the MSISE model (NRLMSISE-00 and MSISE-90) density during strong solar and/or geomagnetic activities. It is well known that there are two major mechanisms to induce satellite drag caused by atmospheric density enhancement: the heating by solar EUV radiation and joule heating associated with local geomagnetic current enhancements during geomagnetic storms. For this work we select five events dominated by the radiation effect and/or the geomagnetic effect. For these events we compared the satellite drag derived density with the MSISE model density. The major results can be summarized as follows. (1) The density predicted from the MSISE models during radiation dominated periods are comparable to the drag derived density but the MSISE model density during strong geomagnetic storms is significantly underestimated when the MSISE model density is compared to the drag derived density, by about two times for the NRLMSISE-00 model. (2) The ratios of the KOMPSAT1 (around $685 \mathrm{~km}$ ) drag derived density to the MSISE model density during a strong geomagnetic storm are abruptly enhanced (up to a factor of about 8 for the MSISE-90 model and about 3 for the NRLMSISE-00 model), which are much larger than previous estimates from low altitude (around $400 \mathrm{~km}$ ) satellites. (3) There is a possible correlation between daily drag enhancement and daily Dst variation. We note that there is a remarkable difference in daily drag enhancement although solar and geomagnetic activities are quite similar to each other. We suggest that such a difference should be explained by the accumulation of solar radiation effect depending on solar activity cycle.
\end{abstract}

Key words: Satellite drag, drag derived density, MSISE-90, NRLMSISE-00, LEO.

\section{Introduction}

It is well known that extreme space environments caused by solar activity cause great effects on satellites in several different ways. Among several effects, we focus on satellite drag caused by the density enhancement of the neutral atmosphere. There are two major physical processes to enhance the neutral atmosphere density: (1) the heating by solar extreme ultra-violet (EUV) radiation, and (2) joule heating associated with local current enhancements during geomagnetic storms. The increased density of the neutral atmosphere is directly proportional to the drag experienced by a low earth orbit (LEO) satellite and the increased drag can, in a matter of tens of minutes to hours, significantly affect satellite orbit parameters (Tobiska, 2002).

The drag acceleration equation of the satellite orbit is given by:

$$
a_{\mathrm{D}}=\frac{1}{2} C_{\mathrm{D}} \frac{A}{M} \rho V_{\mathrm{r}}^{2}
$$

*Now at the Department of Physics, University of New Hampshire, Durham, NH 03824, U.S.A.

Copyright (c) The Society of Geomagnetism and Earth, Planetary and Space Sciences (SGEPSS); The Seismological Society of Japan; The Volcanological Society of Japan; The Geodetic Society of Japan; The Japanese Society for Planetary Sciences; TERRAPUB where $a_{\mathrm{D}}$ is the drag acceleration in a direction opposite to the velocity vector, $C_{\mathrm{D}}$ is the drag coefficient, $A$ is the effective satellite cross sectional area perpendicular to the direction of motion, $M$ is the satellite mass, $\rho$ is the atmospheric density, and $V_{\mathrm{r}}$ is the orbital velocity (Knowles et al., 2001; Montenbruck and Gill, 2001; Marcos and Wise, 2002). If one knows the satellite parameters and drag coefficient, the density of the upper atmosphere can be determined by the orbit information of satellite.

Recently, the density of the upper atmosphere of the Earth has been accurately derived by satellites with high resolution accelerometers, for instance, CHAMP and GRACE. These kinds of works have demonstrated that the Mass-Spectrometer-Incoherent-Scatter (MSIS) model underestimates the atmospheric density during the geomagnetic storms in comparison of the drag derived density (Liu et al., 2005; Liu and Lühr, 2005; Burke et al., 2007). According to Liu et al. (2005), a comparison between observations and the MSISE-90 model predictions showed that although the model described the general structure of the observed density reasonably well, it missed the double peaks at low latitudes completely. This caused an underestimation of the total mass density by about $\sim 20 \%$ in the crest region. At high latitudes an underestimation of $\sim 30 \%$ occurred in the midnight sector and the cusp region. They also investi- 
Table 1. Strong solar and/or geomagnetic events used in our analysis.

\begin{tabular}{|c|c|c|c|c|c|c|c|}
\hline & & Solar & Act. & & & Geomag. & Act. \\
\hline $\begin{array}{l}\text { Event } \\
\text { date }\end{array}$ & $\begin{array}{l}\text { Event } \\
\text { time }\end{array}$ & $\begin{array}{l}\text { Flare } \\
\text { site }\end{array}$ & $\begin{array}{c}\text { Flare } \\
\text { (x-ray) }\end{array}$ & $\begin{array}{c}V_{\mathrm{CME}} \\
(\mathrm{km} / \mathrm{s})\end{array}$ & F10.7 & $\begin{array}{c}\text { Date \& Time } \\
\text { (yyyymmddhh) }\end{array}$ & $\begin{array}{l}\text { Dst } \\
(\mathrm{nT})\end{array}$ \\
\hline 20010329 & $10 \mathrm{~h} 26 \mathrm{~m}$ & N20W19 & $\mathrm{X} 1.7$ & 942 & 274 & 2001033108 & -387 \\
\hline 20011121 & $14 \mathrm{~h} 06 \mathrm{~m}$ & S14W19 & $\mathrm{C} 4.7$ & 518 & 184 & 2001112416 & -221 \\
\hline 20011228 & $20 \mathrm{~h} 30 \mathrm{~m}$ & S26E90 & X3.4 & 2216 & 263 & 2001123005 & -58 \\
\hline 20020720 & $22 \mathrm{~h} 06 \mathrm{~m}$ & SE limb & $\mathrm{X} 3.3$ & 1941 & 185 & 2002072209 & -36 \\
\hline - & - & - & - & - & - & 2002090405 & -109 \\
\hline
\end{tabular}

gated the strong enhancements of the upper thermospheric total mass density during three geomagnetic superstorms in 2003 by using the CHAMP satellite. Liu and Lühr (2005) showed that the MSISE-90 model was unable to reproduce most of the observed features of these three storms and the density was underestimated by the model at all latitudes except for the southern polar regions at the night-side. Burke et al. (2007) compared thermospheric densities predicted by the NRLMSISE-00 model with those inferred from accelerometer measurements by the GRACE satellite during two magnetic storm periods in 2004. They reported that the model significantly under-predicted densities during storms although predictions and measurements were in substantial agreements during quiet times. These recent works demonstrate that the MSISE models are not successful for predicting transient storm-time disturbances.

In this study, we use the drag derived density from the KOrea Multi-Purpose SATellite-1 (KOMPSAT-1), which was launched on December 21, 1999 into a Sunsynchronous circular orbit with an inclination of $98^{\circ}$ and an altitude of $\sim 685 \mathrm{~km}$ (Kim et al., 2004). Recently, Kim et al. (2006) showed that there is one-to-one correspondence between the KOMPSAT-1 drag acceleration and geomagnetic storms: drag acceleration strongly correlates with the level of geomagnetic storms. We present the comparison between the drag derived density of the KOMPSAT1 satellite and the MSISE models such as MSISE-90 and NRLMSISE-00.

Our main interest is summarized as follows. First, as we mentioned before, there are two main origins of satellite drag: solar radiation effect and joule heating associated with geomagnetic storms. We want to examine the dependence of both effects using five events whose solar and/or geomagnetic activities were strong. Second, the altitude of KOMPSAT- 1 is about $685 \mathrm{~km}$, which is much higher than those of previous satellites (e.g., $\sim 400 \mathrm{~km}$ for CHAMP and $\sim 500 \mathrm{~km}$ for GRACE) that have been studied so far. We want to examine the difference between the density from CHAMP and GRACE whose altitudes are about $400 \mathrm{~km}$ and the density from KOMPSAT-1 at a much higher altitude. Third, we investigate the relationship between the daily drag enhancement and daily Dst variation for five events and then compare our results with those for the 10 events used by Kim et al. (2006). Then we discuss what makes a remarkable difference in daily drag enhancement even though solar and geomagnetic activities of two events are nearly similar to each other. The paper is organized as follows. The data analysis is described in Section 2. We present results and discussions in Section 3. A brief sum- mary and conclusion are delivered in Section 4.

\section{Data Analysis}

For the present work, we select five events characterized by strong solar and/or geomagnetic activities. Their basic information is summarized in Table 1. For all these events, there were very strong X-class flares and/or severe geomagnetic storms. The flare information is taken from the NGDC flare list*1 and the CME information from the SOHO/LASCO CME online catalogue*2. We use the daily F10.7 index $^{* 3}$ provided at Space Environment Center. This index has been used as a conventional proxy to represent solar EUV radiation (Donnelly et al., 1983). The Dst (Disturbance storm time) index indicates the enhancement of ring currents by geomagnetic storms and is taken from the World Data Center for Geomagnetism*4.

One of the most important space weather applications is a routine estimation of upper atmospheric drag for precise determination of orbits for satellites in LEO (Nicholas et al., 2000). Drag on LEO satellites is the largest source of error in orbit determination, primarily because of the inaccuracy of upper atmospheric mass density estimates (Marcos et al., 1998). Using the drag acceleration equation given by Eq. (1), we derive the total mass density of the upper atmosphere. In this estimation, we use the average mass of KOMPSAT- 1 of $448 \mathrm{~kg}$, the average cross-section of $5.871 \mathrm{~m}^{2}$, and the velocity of $7.5 \mathrm{~km} / \mathrm{s}$ for $685 \mathrm{~km}$ altitude. According to Knowles et al. (2001), the drag coefficient $C_{\mathrm{D}}$ varies with the flow regime, and is approximately 2.2 for a perfect sphere with the free molecular flow regime. Since KOMPSAT-1 has a shape of hexagon and an average drag coefficient estimated from its long term operation from 2000 to 2002 is around 2.2 (Kim et al., 2004), we assume that $C_{\mathrm{D}}$ is 2.2 .

The orbit determination of KOMPSAT-1 is carried out twice (09:00 and 21:00 UT, 18:00 and 06:00 KST) a day using onboard GPS (Global Positioning System) navigation solution data and its accuracy is estimated to be about $1.6 \mathrm{~m}$ when general acceleration is considered during the period of low solar activity (Kim et al., 2004). The drag-derived density that we use is an average along the satellite path. In this study, we use the 12-hour average drag acceleration data deduced from the change of a semi-major axis, which

\footnotetext{
*1 (ftp://ftp.ngdc.noaa.gov/STP/SOLAR_DATA/SOLAR_FLARES/XRAY_ FLARES).

*2 (http://cdaw.gsfc.nasa.gov/CME_list/index.html).

*3 (http://www.sec.noaa.gov/ftpmenu/indices/old_indices.html).

*4 (http://swdcwww.kugi.kyoto-u.ac.jp).
} 
is determined using an orbit determination program (Kim et al., 2004) based on GPS navigation solution data.

The MSISE models such as MSISE-90 and NRLMSISE00 describe the neutral temperature and densities in the upper atmosphere (above $100 \mathrm{~km}$ ). The data sources of MSISE-90 model include measurements from several rockets, satellites and incoherent scatter radars (Hedin, 1987, 1991) and the data sources of NRLSMSISE-00 also include total mass density from satellite accelerometers as well as orbit determination (Picone et al., 2002). In this study, we use the MSISE-90 model which is available at the online web site ${ }^{* 5}$ and the NRLMSISE-00 model which is taken from the Space Physics Models page of the Goddard Space Flight Center ${ }^{* 6}$. According to Picone et al. (2002), the new model, NRLMSISE-00, handles spatial and temporal variability somewhat better than the MSISE-90 model at elevated geomagnetic activity.

The MSISE models require several input parameters such as input year, day of year, universal time, altitude, geodetic latitude and longitude, local apparent solar time, solar F10.7 flux (for previous day and three-month average), and geomagnetic Ap index (daily or Ap history for the last 59h). The position data (latitude and longitude) of the satellite are also used as input parameters of the model. The geomagnetic 3 hour ap index is used for the MSISE models from the World Data Center for Geomagnetism*7. With the above input parameters, the models produce the following output parameters: number density of $\mathrm{He}, \mathrm{O}, \mathrm{N}_{2}, \mathrm{O}_{2}, \mathrm{Ar}$, $\mathrm{H}$ and $\mathrm{N}$, total mass density, neutral temperature, and exospheric temperature (Hedin, 1987, 1991). The models are computed every six hours (03:00, 09:00, 15:00, 21:00 UT) and then its running average for three values is taken every twelve hours (09:00 and 21:00 UT) for comparison.

\section{Result and Discussion}

For five events in Table 1, we have compared the drag derived density from KOMPSAT-1 with the density from the MSISE models. Figures 1 and 2 show two cases: the first case is the 2001 March 29 event for which the geomagnetic storm effect was quite significant relative to the solar radiation effect and the second case is the 2002 July 20 event for which the radiation effect was dominant. As shown in Table 1, the 2001 March 29 event has not only an X-class flare which enhanced the EUV radiation characterized by F10.7 increase, but also a strong geomagnetic storm with Dst $=-387 \mathrm{nT}$. The drag derived density gradually increases from DOY 85 , which seems to be caused by the increase of F10.7, and then has a local maximum around DOY 87, which is coincident with an intermediate geomagnetic storm. There is a very strong enhancement around DOY 90, which is well consistent with the abrupt decrease of Dst index. As shown in the figure, the variations of drag derived density and Dst have quite similar patterns, implying that the drag derived density is mostly represented by the variation of Dst value. While the MSISE90 model does not account for the density enhancement at

\footnotetext{
*5 (http://modelweb.gsfc.nasa.gov/atmos/msise.html).

*6 (http://modelweb.gsfc.nasa.gov/atmos/nrlmsise00.html).

${ }^{* 7}$ (http://swdcwww.kugi.kyoto-u.ac.jp).
}

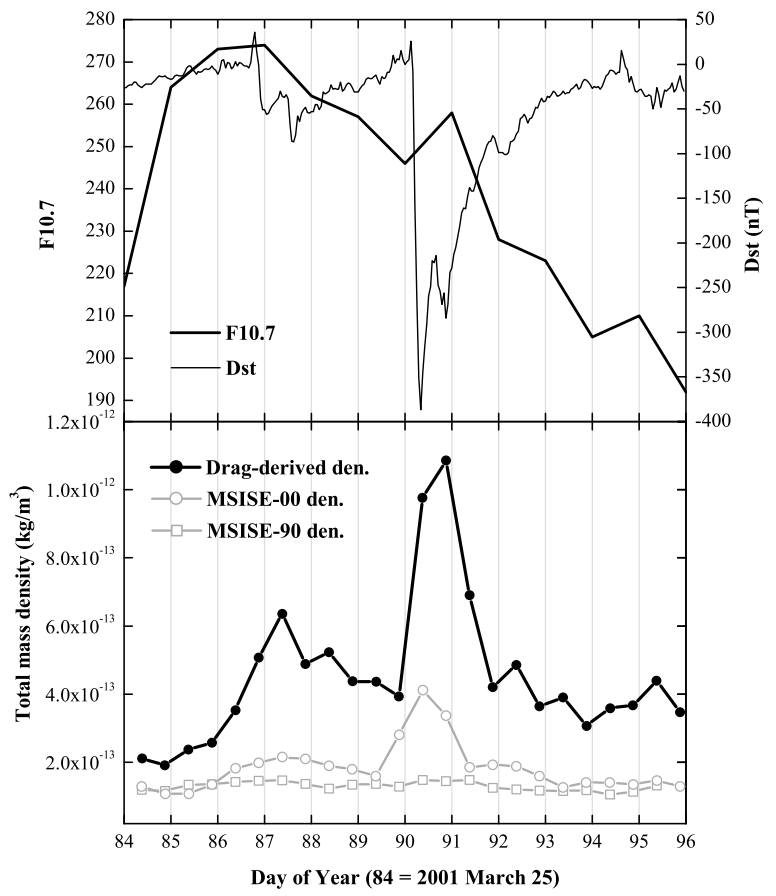

Fig. 1. F10.7 (thick solid line) and Dst (thin solid line) indices from March 25 (DOY 84) to April 5, 2001 (top). Comparison between the drag derived density (filled circle) and the density from the NRLMSISE-00 model (gray open circle) and the density from the MSISE-90 model (gray open square) for 2001 March 29 event (bottom).

all, the NRLMSISE-00 model gives about three times enhancement, which is still underestimated to match the drag derived density by a factor of about 3. Burke et al. (2007) reported that the NRLMSISE-00 model under-predicted the density from GRACE accelerometer during geomagnetic storms by a factor of 1.5 (for details, see their Fig. 2). According to Liu and Lühr (2005), the drag derived density from CHAMP ( $400 \mathrm{~km}$ altitude) is about 5 times higher than that from the MSISE-90 model at high geomagnetic latitudes during a strong geomagnetic storm. In our case, the ratio of drag derived density to the MSISE-90 model density is up to 8 during the 2001 March 29 event which has the strongest geomagnetic storm among our samples.

For the 2002 July 20 event, there is no geomagnetic storm. Figure 2 shows that the drag derived density and MSISE model density are comparable to each other and their patterns are quite similar. These facts demonstrate that the MSISE models well reflect the variation of atmospheric density caused by solar EUV radiation. The F10.7 index gradually increases with time and its variation is quite similar to that of the KOMPSAT- 1 drag derived density. A cross-correlation between the F10.7 index and the drag derived density has a maximum $(r=0.92)$ at 1 -day time delay, which is consistent with Jacchia et al. (1973) who found a lag of approximately 1 day between solar activity and atmospheric densities derived from the drag of 10 satellites in the interval 1958-1971. Our results from both cases (Figs. 1 and 2) show that the MSISE models do not properly account for the density enhancement caused by strong geomagnetic storms at a high altitude (around $690 \mathrm{~km}$ ), which is also consistent with recent studies (e.g., Liu et al., 2005; 


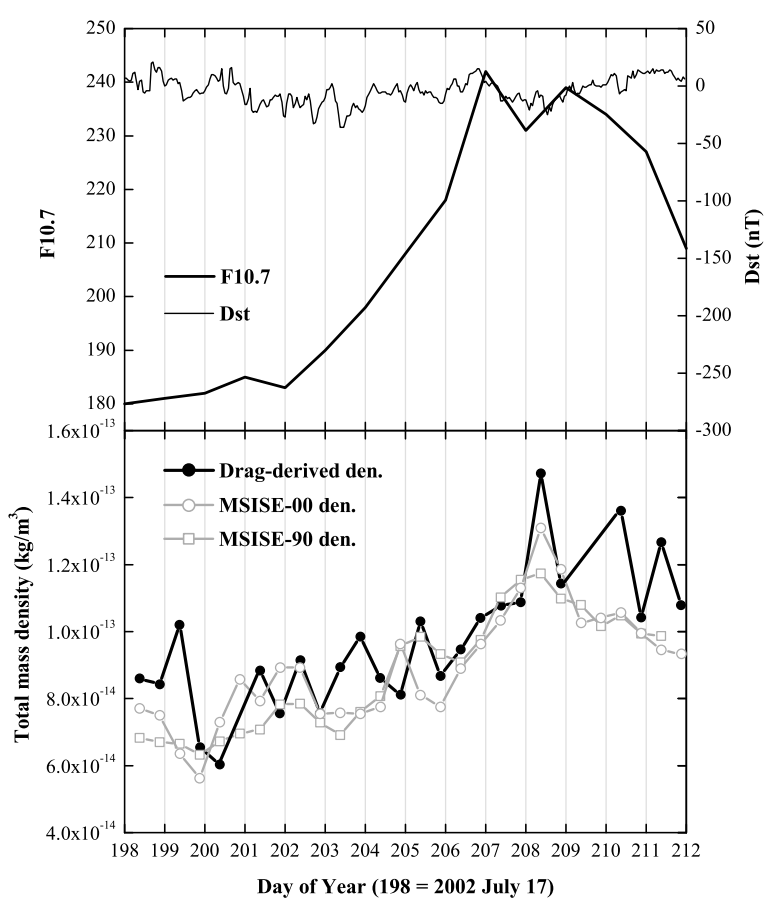

Fig. 2. F10.7 (thick solid line) and Dst (thin solid line) indices from July 17 (DOY 198) to 30, 2002 (top). Comparison between the drag derived density (filled circle) and the NRLMSISE-00 model (gray open circle) and the density from the MSISE-90 model (gray open square) for 2002 July 20 event (bottom).

Liu and Lühr, 2005; Burke et al., 2007) for low altitudes (around $400 \mathrm{~km}$ ). However, we note that the density from the NRLMSISE-00 agrees better with the drag derived one than that from the MSISE-90 model at the high altitude during strong geomagnetic storms. Regarding this issue, our results support an argument that the current atmospheric models are statistical and do not allow a short-term prediction for a given altitude (Koskinen et al., 2001).

Figure 3 shows the ratio of the drag derived density to the MSISE model density as a function of day for all five events summarized in Table 1. The 2001 November 21 event that was associated with a weak C-class flare, produced a strong geomagnetic storm $(-221 \mathrm{nT})$. The 2001 December 28 event has a very strong X3.4 flare and a relatively large value (263) of F10.7 index. It produced a weak geomagnetic storm $(-58 \mathrm{nT})$. In the case of the 2002 September 4 event, there was only a moderate geomagnetic storm (Dst $=-109 \mathrm{nT}$ ). A detailed investigation of solar wind data (not shown here) shows that this event seems to be associated with corotating interaction regions (CIRs) caused by high speed stream (Mullan, 1984). For these five events, we found a couple of interesting results as follows. (1) The ratio between the drag derived density and NRLMSISE-00 is quite similar to that between the drag derived density and the MSISE-90 model during non-storm times but obviously smaller during geomagnetic storms. As shown in the top panel of Fig. 3, while the ratio of the drag derived density and the MSISE-90 model is 7.5 during a strong geomagnetic storm, the ratio between the drag derived density and NRLMSISE-00 is 3.2. We found a tendency that the bigger the storm, the higher the ratio. (2) The ratios during non-

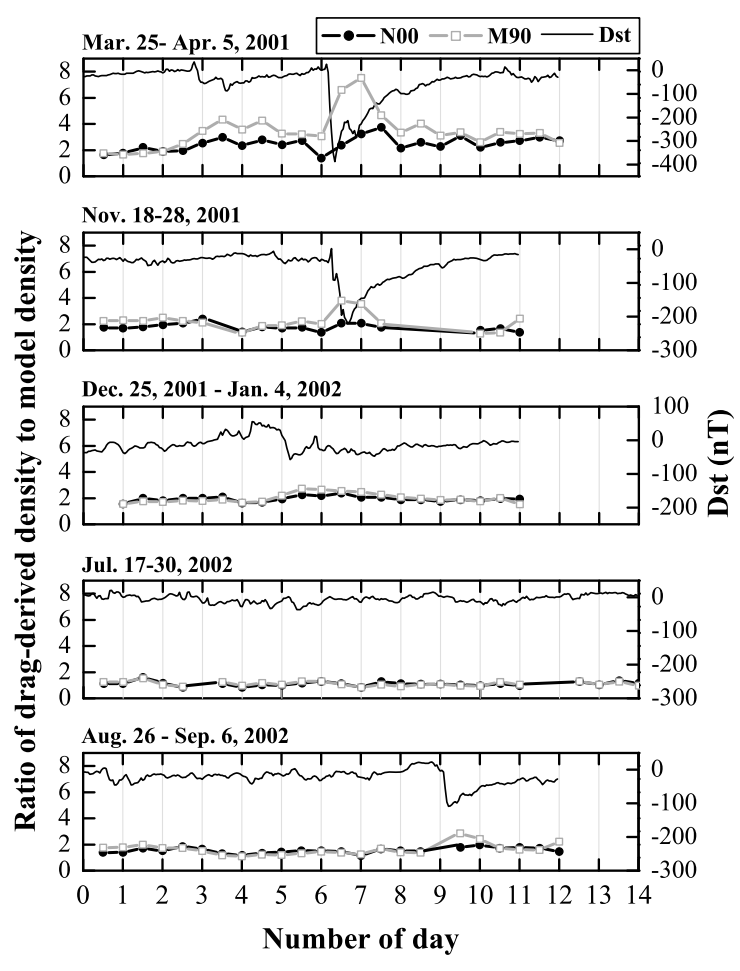

Fig. 3. Ratio of the drag derived density to the NRLMSISE-00 density (filled circle) and to the MSISE-90 density (open square) as a function of day with Dst index (thin solid line) for five events. storm times slightly increase with long-term solar activity. While two events (20020720 and 20020904) in 2002 have the two lowest ratios (about 1.2 on the fourth day), which are thought to be probably the background values during non-storm times, the other three events in 2001 have larger background values (around 2). Table 2 summarizes the ratio of the drag derived density to the MSISE model density from previous publications and the present study. The comparison shows that the ratio generally increases with geomagnetic storm strength and altitude. It is not so clear to argue the dependence of the ratio on F10.7. For example, a comparison between the 20010328 and 20011230 events in Table 2 shows that both ratios of 20010328 are about 1.5 times larger than those of 20011230 even though solar and geomagnetic indices are nearly the same. This difference may be explained by other effects such as long-term solar radiation effect.

For a quantitative comparison of the tendency, we compare the daily Dst variation with the daily drag enhancement for five events in Fig. 4. In addition, we include the KOMPSAT-1 drag data (open circle) during three month period including the Halloween event (October-December, 2003). During the period, there were two very strong geomagnetic storms such as the October 29-30 event and the November 20 event as well as several weak or moderate Dst enhancements. For these 10 events, Kim et al. (2006) showed that drag acceleration correlates strongly with the level of geomagnetic disturbance $(r=-0.95)$. In Fig. 4, one may find two different slopes between daily Dst variation and daily drag enhancement: one slope for 2001 year events and the other for the remaining events. Especially, 
Table 2. Summary of the comparison between the drag derived density $\left(\rho_{\mathrm{d}}\right)$ to the MSISE model ${ }^{\mathrm{a}}$ density $\left(\rho_{\mathrm{m}}\right)$ for eight geomagnetic storms, which are taken from previous publications and the present study.

\begin{tabular}{cccccccc}
\hline Date & F10.7 & Dst & Satellite & Height & Model $^{\mathrm{a}}$ & $\rho_{\mathrm{d}} / \rho_{\mathrm{m}}$ \\
\hline 19830724 & 136 & $-74 \mathrm{nT}$ & SETA & $\sim 200 \mathrm{~km}$ & M90 & 1.5 & Forbes et al. $(1996)$ \\
20031120 & 175 & $-472 \mathrm{nT}$ & CHAMP & $\sim 400 \mathrm{~km}$ & M90 & 5.0 & Liu and Lühr $(2005)$ \\
20041108 & 124 & $-373 \mathrm{nT}$ & GRACE & $\sim 350 \mathrm{~km}$ & N00 & 1.5 & Burke et al. $(2007)$ \\
20010331 & 246 & $-387 \mathrm{nT}$ & KOMPSAT-1 & $\sim 695 \mathrm{~km}$ & M90/N00 & $7.5 / 3.2$ & This study \\
20010328 & 274 & $-56 \mathrm{nT}$ & KOMPSAT-1 & $\sim 695 \mathrm{~km}$ & M90/N00 & $4.3 / 3.0$ & This study \\
20011124 & 173 & $-221 \mathrm{nT}$ & KOMPSAT-1 & $\sim 693 \mathrm{~km}$ & M90/N00 & $3.8 / 2.1$ & This study \\
20011230 & 247 & $-58 \mathrm{nT}$ & KOMPSAT-1 & $\sim 690 \mathrm{~km}$ & M90/N00 & $2.7 / 2.3$ & This study \\
20020904 & 171 & $-109 \mathrm{nT}$ & KOMPSAT-1 & $\sim 685 \mathrm{~km}$ & M90/N00 & $2.9 / 1.8$ & This study \\
\hline
\end{tabular}

${ }^{\mathrm{a} M 90,}$ MSISE-90; N00, NRLMSISE-00.

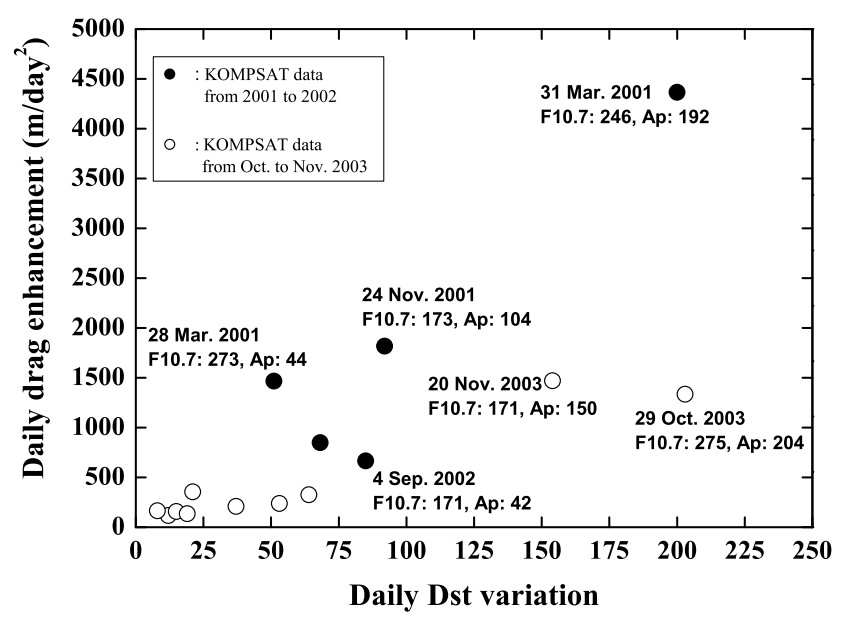

Fig. 4. Comparison of the daily Dst variation and the daily drag enhancement. Both quantities correspond to the absolute difference between the perturbed and the background. Filled circles indicate the KOMPSAT-1 data from 2001 to 2002 and open circles represent those used by Kim et al. (2006), respectively. F10.7 and Ap index for several strong events are shown to indicate how much there were strong solar and geomagnetic activities.

we note that there is a remarkable difference in daily drag enhancement between the 2001 March 29 event and the 2003 October 29 event. In these two cases, F10.7, Dst, and Ap index are all quite similar to each other so that we may expect similar drag enhancements. However, the drag enhancement of the first event is about three times larger than that of the second event. This fact implies that there should be another important factor to control the satellite drag. As a possible factor, we suggest the accumulation effect of solar EUV radiation. Figure 5 shows the long-term variation of F10.7 index of these two events for one year. It is noted that the solar EUV radiation in 2001 is remarkably higher than that in 2003. This fact implies that the neutral atmosphere in 2001 be already more expanded relative to that in 2003 due to long term solar radiation effect.

\section{Summary and Conclusion}

In this study, we have compared the drag derived density from the KOMPSAT-1 satellite with the empirical MSISE models such as NRLMSISE-00 and MSISE-90 during strong solar and/or geomagnetic activities. The major results can be summarized as follows. (1) The density predicted from the MSISE models are similar to the drag de-

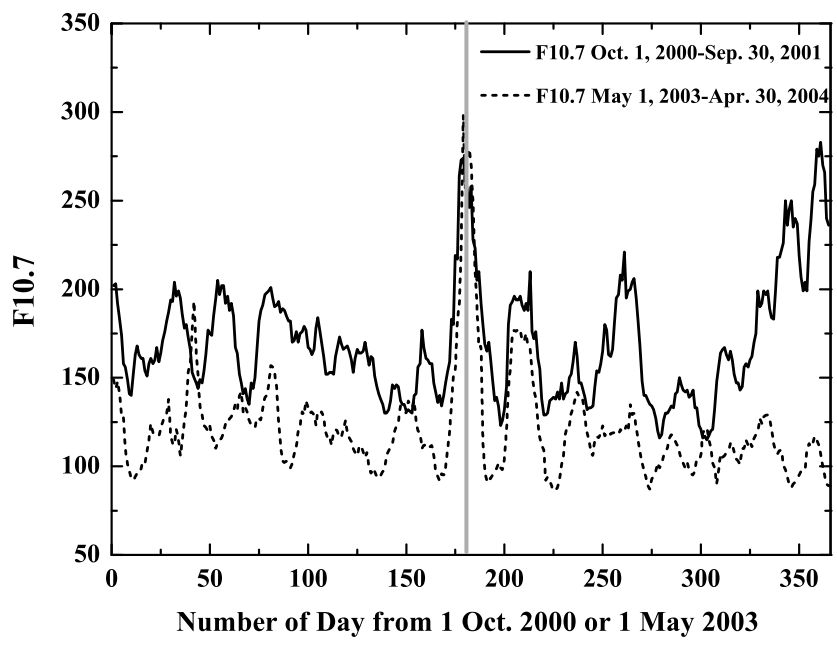

Fig. 5. Long-term variation of F10.7 index for two events (2001 March 29 and 2003 October 29). The solid line starts from Oct. 2000 and the dashed line from May 2003. The vertical line indicates the event days on which strong geomagnetic storms occurred.

rived density during the radiation dominated periods. However, the density from the MSISE models is significantly underestimated when compared to the drag derived density, by about two times for the NRLMSISE-00 model depending on solar activity. (2) The ratios of the KOMPSAT-1 (around $685 \mathrm{~km}$ ) drag derived density to the MSISE model density during a strong geomagnetic storm are abruptly enhanced, for example, up to a factor of about 8 for the MSISE-90 model and about 3 for the NRLMSISE-00 model. These values are much larger than previous estimates (e.g., Liu et al., 2005; Liu and Lühr, 2005; Burke et al., 2007) from low altitude (around $400 \mathrm{~km}$ ) satellites. (3) There is a remarkable difference in daily drag enhancement although solar and geomagnetic activities are quite similar. Such a difference may be explained by the accumulation of long-term solar radiation effect.

It was found from the first and second results that the MSISE models do not properly reflect the variation of atmospheric density during the strong geomagnetic storms. As mentioned above, the MSISE models are empirical models formed by using the data from several rockets, satellites, and incoherent scatter radars (Hedin, 1987, 1991) and the data sources of NRLSMSISE-00 also include total mass density from satellite accelerometers and orbit determina- 
tion (Picone et al., 2002). The MSISE models use several input parameters such as input year, day of year, universal time, altitude, latitude, longitude, local apparent solar time, solar F10.7 flux, and geomagnetic Ap index. To be a successful empirical model, there should be enough observed data to show the dependence on each variable. However, a geomagnetic storm intermittently occurred during a relatively short period (several hours to a few days) so that it is difficult to have enough data for given each different input variable. In another aspect, the model may not reflect the density variation at high altitudes because the study of satellite drag in this position has not been limited. According to Hedin (1987), the data become very sparse in the exosphere about $600 \mathrm{~km}$ and upward extrapolations are expected to become increasingly inaccurate at higher altitudes. Although the NRLMSISE-00 database include a new component, "anomalous oxygen", with appreciable $\mathrm{O}^{+}$and hot atomic oxygen contributions to the total mass density at high altitudes larger than $500 \mathrm{~km}$ (Picone et al., 2002), the NRLMSISE-00 model is still underestimated when compared to the drag derived density. We also note from the third result that the long-term solar radiation effect is not properly included in the MSISE models. According to Hedin (1983), the remaining area of poorest data coverage at satellite altitudes is at high latitudes for high solar activity. From the present study, we found the importance of the combination effect between long-term solar radiation effect and geomagnetic effect as shown in Figs. 4 and 5. A further detailed examination is desirable for improving the atmospheric models as well.

To improve an empirical atmospheric model, we have a few suggestions as follows. First, there should be sufficient satellite drag data covering different input variables and different solar cycles. Especially, the present study shows that high altitude LEO satellite data and long-term radiation effect are important to improve the model. Second, we should try to find a better input parameter for the model. Regarding this issue, Burke et al. (2007) showed from GRACE observations that polar cap potential led the drag derived density from GRACE satellite by $\sim 4$ hours during stormtime. Third, we need more detailed comparisons between the drag derived density and empirical models. In this respect, the GRACE and CHAMP data are invaluable in that they make it possible to directly estimate the drag derived density using accelerometer. Finally we briefly discuss the limitation of the present study. We used the KOMPSAT-1 satellite drag acceleration data based on the GPS data with twelve hours time resolutions. Even though our data have two disadvantages (measurement method and time resolution) over CHAMP and GRACE data, our results are consistent with recent results and also give us several important implications on the improvement of empirical atmospheric models such as MSISE.

Acknowledgments. We really appreciate the referees' constructive comments. We are very thankful to Drs Thomson, Burke and Liu for their helpful discussions. This work has been supported by the MOST grants (M1-0104-00-0059 and M1-0407-00-0001), the Korea Research Foundation (KRF-2005-070-C00059), and the KASI (Korea Astronomy and Space Science Institute) basic research fund of the Korean government.

\section{References}

Burke, W. J., C. Y. Huang, F. A. Marcos, and J. O. Wise, Interplanetary Contral of Thermospheric Densities during Large Magnetic Storms, $J$. Atmos. Solar-Terr. Phys., 69, 279-287, 2007.

Donnelly, R. F., D. F. Heath, J. L. Lean, and G. J. Rottman, Differences in the temporal variations of solar UV flux, $10.7-\mathrm{cm}$ solar radio flux, sunspot number, and $\mathrm{Ca}-\mathrm{K}$ plage data caused by solar rotation and active region evolution, J. Geophys. Res., 88, 9883-9888, 1983.

Forbes, J. M., R. Gonzalez, F. A. Marcos, D. Revelle, and H. Parish, Magnetic storm response of lower thermosphere density, J. Geophys. Res., 101, 2313-2320, 1996.

Hedin, A. E., A revised thermospheric model based on mass Spectrometer and incoherent scatter data: MSIS-83, J. Geophys. Res., 88, 10170$10188,1983$.

Hedin, A. E., MSIS-86 thermospheric model, J. Geophys. Res., 92, 46494662, 1987.

Hedin, A. E., Extension of the MSIS thermosphere model into the middle and lower atmosphere, J. Geophys. Res., 96, 1159-1172, 1991.

Jacchia, L. G., J. W. Slowley, and I. G. Campbell, An analysis of the solaractivity effects in the upper atmosphere, Planet. Space Sci., 21, 18351842, 1973.

Kim, H.-D., E.-K. Kim, and H.-J. Choi, Orbit determination for the KOMPSAT-1 Spacecraft during the period of the solar maximum, KSAS International Journal, 6, 71-84, 2004.

Kim, K.-H., Y.-J. Moon, K.-S. Cho, H.-D. Kim, and J.-Y. Park, Atmospheric drag effects on the KOMPSAT-1 satellite during geomagnetic superstorms, Earth Planets Space, 58, e25-e28, 2006.

Knowles, S. H., J. M. Picone, S. E. Thonnard, and A. C. Nicholas, The Effect of Atmospheric Drag on Satellite Orbits During the Bastille Day Event, Solar Physics, 204, 387-397, 2001.

Koskinen, H., E. Tanskanen, R. Pirjola, A. Pulkkinen, C. Dyer, D. Rodgers, P. Cannon, J.-C. Mandeville, and D. Boscher, Space weather effects catalogue, ESA Space Weather Study in pressed, 2001.

Liu, H. and H. Lühr, Strong disturbance of the upper thermospheric density due to magnetic storms: CHAMP observations, J. Geophys. Res., 110, CiteID A09S29, doi:10.1029/2004JA010908, 2005.

Liu, H., H. Lühr, V. Henize, and W. Köhler, Global distribution of the thermospheric total mass density derived from CHAMP, J. Geophys. Res., 110, CiteID A04301, doi:10.1029/2004JA010741, 2005.

Marcos, F. A. and J. O. Wise, Towards a golden age of satellite drag, AIAA Aerospace Sciences Meeting and Exhibit, 40th, Reno, NV, Jan. 14-17, AIAA-2002-92, 2002.

Marcos, F. A., M. J. Kendra, J. M. Griffen, J. N. Bass, D. R. Larson, and J. F. Lui, Precision Low Earth Orbit Determination Using Atmospheric Density Calibration, Advances in the Astronautical Sciences, 97, 501513, 1998.

Montenbruck, O. and E. Gill, Satellite orbits: Models, methods, and applications, 83 pp., Springer Press., Berlin, 2001.

Mullan, D. J., Corotating interaction regions in stellar winds, Astrophysical Journal, 283, 303-312, 1984.

Nicholas, A. C., J. M. Picone, S. E. Thonnard, R. R. Meier, K. F. Dymond, and D. P. Drob, A methodology for using optimal MSIS parameters retrieved from SSULI data to compute satellite drag on LEO objects, J. Atmos. Solar-Terr. Phys., 62, 1317-1326, 2000.

Picone, J. M., A. E. Hedin, D. P. Drob, and A. C. Aikin, NRLMSISE00 empirical model of the atmosphere: Statistical comparisons and scientific issues, J. Geophys. Res., 107, CiteID 1468, doi:10. 1029/2002JA009430, 2002.

Tobiska, W. K., E10.7 Use for Global Atmospheric Density Forecasting in 2001, AIAA/AAS Astrodynamics Specialist Conference and Exhibit, Monterey, California, Aug. 5-8, AIAA-2002-4892, 2002.

J. Park (e-mail: jdu35@unh.edu), Y.-J. Moon (e-mail: moonyj@khu. ac.kr), K.-H. Kim, K.-S. Cho, H.-D. Kim, Y.-S. Kwak, Y.-H. Kim, Y.-D. Park, and Y. Yi 